\title{
Spin-resolved spectra of Shiba multiplets from Mn impurities in MgB2
}

\section{Citation}

Moca, Catalin Pascu, Eugene Demler, Boldizsár Jankó, and Gergely Zaránd. 2008. Spin-Resolved Spectra of Shiba Multiplets from Mn Impurities in MgB2. Physical Review B 77 (17). doi:10.1103/ physrevb.77.174516.

\section{Published Version}

doi:10.1103/PhysRevB.77.174516

\section{Permanent link}

http://nrs.harvard.edu/urn-3:HUL.InstRepos:27889189

\section{Terms of Use}

This article was downloaded from Harvard University's DASH repository, and is made available under the terms and conditions applicable to Other Posted Material, as set forth at http:// nrs.harvard.edu/urn-3:HUL.InstRepos:dash.current.terms-of-use\#LAA

\section{Share Your Story}

The Harvard community has made this article openly available.

Please share how this access benefits you. Submit a story.

Accessibility 


\title{
gु \\ Spin-resolved spectra of Shiba multiplets from $\mathrm{Mn}$ impurities in $\mathrm{MgB}_{2}$
}

\author{
Cătălin Paşcu Moca, ${ }^{1,2}$ Eugene Demler, ${ }^{3}$ Boldizsár Jankó, ${ }^{4,5}$ and Gergely Zaránd ${ }^{1}$ \\ ${ }^{1}$ Budapest University of Technology and Economics, H-1521 Budapest, Hungary \\ ${ }^{2}$ Department of Physics, University of Oradea, Oradea 410087, Romania \\ ${ }^{3}$ Department of Physics, Harvard University, 17 Oxford Street, Cambridge, Massachusetts 02138, USA \\ ${ }^{4}$ Materials Science Division, Argonne National Laboratory, 9700 South Cass Avenue, Argonne, Illinois 60439, USA \\ ${ }^{5}$ Department of Physics, University of Notre Dame, Notre Dame, Indiana 46556, USA
}

(Received 18 March 2008; published 30 May 2008)

\begin{abstract}
We study the effect of magnetic $\mathrm{Mn}$ ions on the two-band superconductor $\mathrm{MgB}_{2}$, and compute both the total and spin-resolved scanning tunneling spectrum in the vicinity of the magnetic impurity. We show that when the internal structure of the Mn ion's $d$-shell is taken into account, multiple Shiba states appear in the spectrum. The presence of these multiplets could alter significantly the overall interpretation of local tunneling spectra for a wide range of superconducting hosts and magnetic impurities.
\end{abstract}

DOI: 10.1103/PhysRevB.77.174516

PACS number(s): 74.25.Jb

\section{INTRODUCTION}

The interaction between a single magnetic impurity and the superconducting host reveals fundamental properties of both the magnetic ion and the host material. This interaction was first studied theoretically, within the framework of BCS superconductivity. During the late sixties, Shiba ${ }^{1}$ showed that a magnetic impurity pulls down from the continuum states a pair of bound states inside the superconducting gap. Indirect indication for the presence of finite spectral weight inside the gap of an impure superconductor could be inferred from global probes of the density of states. However, direct evidence for the existence of the so-called Shiba states requires an accurate measurement of the local density of states near the impurity. Such a measurement became available only recently by using high vacuum, low temperature scanning tunneling spectroscopy (STS). Yazdani et al. ${ }^{2}$ imaged the local density of states around $\mathrm{Mn}$ and Gd impurities deposited onto $\mathrm{Nb}$ single crystals. They found clear evidence for localized states in the vicinity of the magnetic impurities, in qualitative agreement with Shiba's original findings, and also with their own model calculation based on a non-self-consistent solution to Bogoliubov-de Gennes equations. Quantitative discrepancies, however, are also clearly present, especially when comparing the width and spatial dependence of the resonances to theoretical expectations. The presence of magnetic impurity induced bound states in a superconductor was turned around and used, both theoretically ${ }^{3-5}$ and experimentally, ${ }^{6}$ as an investigative tool to probe the unusual ground state of the cuprate superconductors.

Although there exist some precious numerical renormalization group and Monte Carlo results for quantum dots attached to superconducting electrodes, ${ }^{7,8}$ most of the theoretical studies carried out so far for magnetic impurities in a superconductor follow Shiba's original work, and use predominantly a classical spin model to describe the magnetic impurity and assume a single spin one-half electron channel that couples to the magnetic impurity. Furthermore, the coupling is assumed to be in the $s$-wave channel, and spin-orbit coupling is generally ignored. This set of approximations worked beautifully for most of the experiments performed so far and provided simple, elegant, and intuitive results. However, recent advances in the resolution, stability, and processing of scanning tunneling imaging opened the door for visualizing structures that go beyond the class of Shiba-like models. Indeed, magnetic impurities have a more complicated internal structure: ${ }^{9}$ The magnetic moments are usually due to low-lying and crystal-field split $d$ or $f$ levels with multiple occupancy. The aim of this paper is to demonstrate that (i) the internal structure of the Mn impurity has a major impact on the structure of the Shiba states, and (ii) these features should be readily observable with the current resolution of STS measurements. In particular, multiple channels of charge carriers couple to the magnetic impurity through channel-dependent coupling. The combination of these ingredients generally leads to the appearance of multiple pairs of Shiba states. We compute the spatial and spin structure of the scanning tunneling microscopy (STM) spectra around the magnetic impurity and show that these states appear as distinct resonances inside the superconducting gap, and can be most clearly resolved in spin-resolved STM spectra.

In the following, we illustrate our results on the specific case of $\mathrm{Mn}$-doped $\mathrm{MgB}_{2}$, but we wish to emphasize that much of our discussions carry over to other systems as well, ${ }^{10}$ and that our conclusions are rather general. There are several reasons to choose the $\mathrm{Mn}-\mathrm{MgB}_{2}$ system. Despite the relatively recent discovery of its essentially conventional superconducting phase, $\mathrm{MgB}_{2}$ has been thoroughly characterized both experimentally and theoretically, ${ }^{11}$ and therefore provides an ideal testing ground for our theoretical framework. Several materials parameters of $\mathrm{MgB}_{2}$ are also in a convenient range for our investigation. First, in order to observe a Shiba state by scanning tunneling spectroscopy, one needs a relatively large gap. $\mathrm{MgB}_{2}$ is a perfect candidate in this respect since it is a conventional superconductor that has an unusually high critical temperature, ${ }^{12} T_{c}=39 \mathrm{~K}$. Second, $\mathrm{MgB}_{2}$ has a hexagonal $\mathrm{AlB}_{2}$-type structure and a highly anisotropic band structure. ${ }^{13,14}$ As we shall see below, this leads to a clear separation of the multiple Shiba states. The presence of two gaps in $\mathrm{MgB}_{2}$ has been well established by now through a variety of spectroscopic probes. ${ }^{15-18}$ It is therefore an interesting question how the presence of these two gaps 
influences the structure of Shiba states. Although a series of experimental $^{19,20}$ and theoretical ${ }^{21,22}$ investigations have been recently completed for $\mathrm{MgB}_{2}$ doped with nonmagnetic as well as magnetic impurities, no experimental or theoretical study has been completed for the local electronic structure of a single magnetic impurity in this compound. This paper now provides a detailed theoretical discussion of the single magnetic impurity problem in $\mathrm{MgB}_{2}$ and other superconductors where the multiple degrees of freedom of the conduction electrons and the impurity could lead to experimentally observable consequences.

Finally, there is another advantage for studying Shiba states in $\mathrm{MgB}_{2}$. The strong coupling, short coherence length, and the consequently robust condensate allow us to investigate the effect of a single magnetic impurity on a superconductor in the regime where the order parameter remains spatially constant. Here, the results of Flatté and Reynolds ${ }^{4}$ are quite valuable: According to their calculations, for a superconductor with coherence length $\xi k_{F}=10$, the relative spatial fluctuations in the local order parameter remain below $5 \%$, even at the impurity site. This result is valid for the entire range of interest $0 \leq g \leq 1$ for the dimensionless coupling $g$ (between the magnetic impurity and the superconducting quasiparticles, see below). As shown in the following sections of this paper, the spatially constant order parameter provides considerable simplifications in our calculations, and this model allows us to make experimentally testable predictions for the presence of the multiple Shiba states in $\mathrm{MgB}_{2}$.

\section{HAMILTONIAN}

\section{A. Band structure calculation}

As mentioned above, $\mathrm{MgB}_{2}$ crystallizes in the hexagonal $\mathrm{AlB}_{2}$-type structure ${ }^{13}$ in which the $B^{-}$ions constitute graphitelike sheets in the form of honeycomb lattices separated by hexagonal layers of $\mathrm{Mg}$ ions. Band structure calculations ${ }^{14}$ indicate that $\mathrm{Mg}$ is substantially ionized, and the bands at the Fermi level derive mainly from boron $p$ orbitals. Four of the six $p$ bands cross the Fermi energy, and the Fermi surface consists of quasi-two-dimensional cylindrical sheets, due to $\mathrm{B}-p_{x, y}$ orbitals, and a three-dimensional tubular network (mostly originating from B- $p_{z}$ orbitals). It is believed that both structures participate in the formation of the superconducting state, although the gap is very different on the tubular network and on the cylindrical sheets.

Let us first discuss the tight-binding Hamiltonian we use and the corresponding band structure. In spite of its simplicity, this tight-binding description is rather robust, as can be checked by a direct comparison to the results of more sophisticated $a b$ initio band structure and density of states (DOS) calculations. ${ }^{14}$ In the rest of the paper, we shall use the following simple Hamiltonian to describe the normal state of $\mathrm{MgB}_{2}$,

$$
H_{0}=\sum_{\mathbf{r}, \mathbf{r}^{\prime}} \sum_{\alpha, \alpha^{\prime}, \sigma}\left(t_{\mathbf{r}, \mathbf{r}^{\prime}}^{\alpha, \alpha^{\prime}}-\mu \delta_{\mathbf{r}, \mathbf{r}^{\prime}} \delta^{\alpha, \alpha^{\prime}}\right)\left(\Psi_{\mathbf{r}, \alpha, \sigma}^{\dagger} \Psi_{\mathbf{r}^{\prime}, \alpha^{\prime}, \sigma}+\text { H.c. }\right),
$$

where $\mu$ sets the Fermi energy and $\Psi_{\mathbf{r}, \alpha, \sigma}$ is the annihilation operator of an electron of spin $\sigma$ on $p$-orbital $\alpha$ ( $\alpha$ $\left.=p_{x}, p_{y}, p_{z}\right)$ of the $\mathrm{B}$ ion at position $\mathbf{r}$,

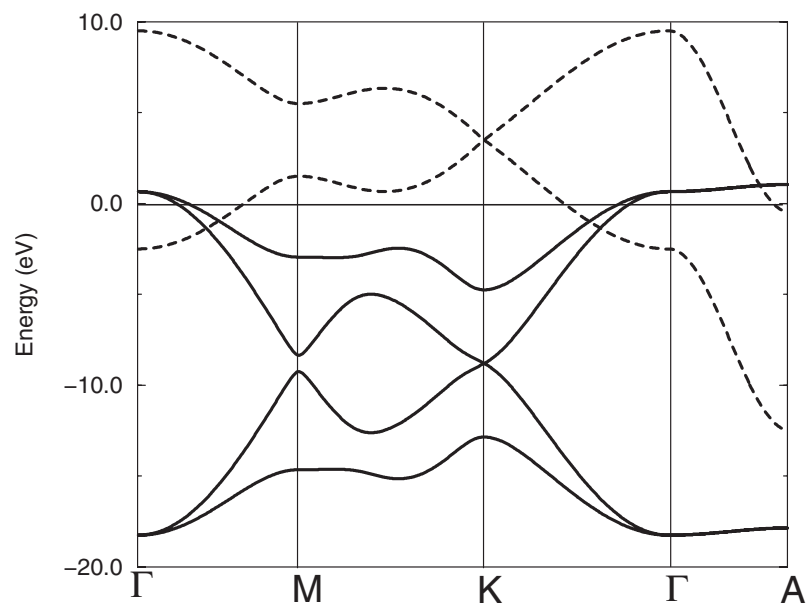

FIG. 1. Band structure for $\mathrm{MgB}_{2}$, along the symmetry lines, computed in the framework of tight-binding model described in Appendix A. There are six bands: four from $p_{x, y}$ orbitals (solid lines) and two from $p_{z}$ (dashed lines). Both $p_{z}$ bands and only two $p_{x, y}$ band cross the Fermi level, which corresponds to zero energy, $E_{F}=0$.

$$
\mathbf{r}=\mathbf{R}+\mathbf{d}
$$

The vector $\mathbf{R}$ in this expression points to the center of the unit cell, and $\mathbf{d}$ gives the position of the $\mathrm{B}$ ion within the unit cell. Note that there are two atoms per unit cell, which shall be labeled by the index $\delta=1,2$ in what follows. The hopping matrix elements $t_{\mathbf{r}, \mathbf{r}^{\prime}}^{\alpha, \alpha^{\prime}}$ in Eq. (1) connect only neighboring sites, but their value depends on the relative orientation of the $p$ orbitals. Quasiparticle energies are measured from the Fermi energy $\mu$.

The Hamiltonian above can be easily diagonalized in Fourier space. The field operators $\Psi_{\mathbf{r}, \alpha, \sigma}$ can be expanded as

$$
\Psi_{\mathbf{r}, \alpha, \sigma}=\frac{1}{\sqrt{\Omega}} \sum_{\mathbf{k}, b} e^{i \mathbf{k} \mathbf{R}} e_{b ; \alpha, \delta}(\mathbf{k}) c_{\mathbf{k}, b, \sigma}
$$

where $\Omega$ is the number of unit cells and $c_{\mathbf{k}, b, \sigma}$ is the annihilation operator of an electron in band $b(b=1, \ldots, 6)$ with momentum $\mathbf{k}$, spin $\sigma$, and energy $\varepsilon_{\mathbf{k}, b}$. The band energies and the wave function amplitudes $e_{b ; \alpha, \delta}$ are determined by the eigenvalue equation

$$
\sum_{\alpha^{\prime}, \delta^{\prime}} H_{\alpha, \delta ; \alpha^{\prime}, \delta^{\prime}}(\mathbf{k}) e_{b ; \alpha^{\prime}, \delta^{\prime}}=\varepsilon_{\mathbf{k}, b} e_{b ; \alpha, \delta}
$$

where $H_{\alpha, \delta ; \alpha^{\prime}, \delta^{\prime}}(\mathbf{k})$ is essentially the Fourier transform of the hopping matrix, detailed in Appendix A. In our tight-binding model, we have six bands: Four of them derive from $p_{x y}$ orbitals while the remaining two from $p_{z}$ orbitals. The band structure obtained is presented in Fig. 1. Notice that both $p_{z}$ ( $\pi$ bands) cross the Fermi surface but only two of the $p_{x, y}$ bands ( $\sigma$ bands) cross it.

In the presence of superconducting order, one must modify the Hamiltonian above and add the pairing terms 


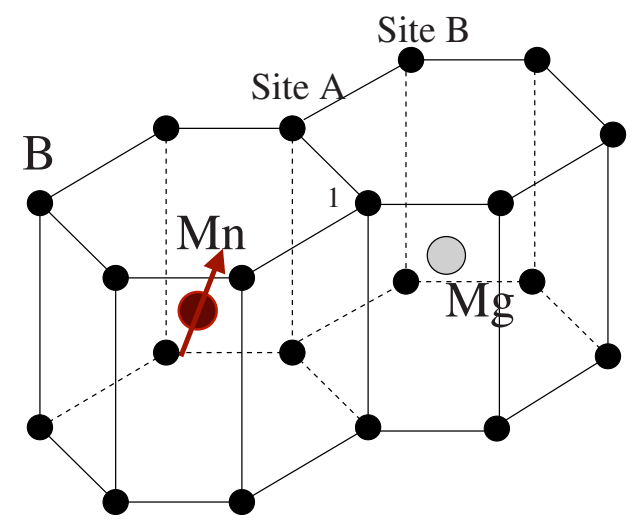

(a)

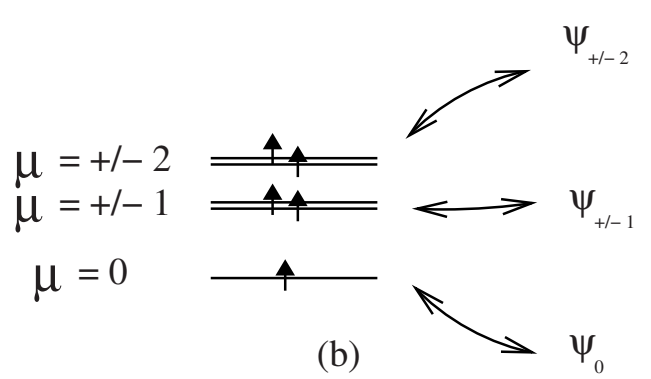

FIG. 2. (Color online) (a) Local environment of an Mn ion in $\mathrm{MgB}_{2}$. The $\mathrm{Mn}$ ion is located in a hexagonal cage made of $\mathrm{B}$ ions represented by large dots. (b) Level structure and crystal-field splitting of the $\mathrm{Mn}^{2+}$ core states. Two levels $(\mu= \pm 2$ and $\mu= \pm 1)$ are twofold degenerate and the level $\mu=0$ is nondegenerate.

$$
H_{0} \rightarrow H_{0}=\sum_{b, \mathbf{k}, \sigma} \varepsilon_{\mathbf{k}, b} c_{\mathbf{k}, b, \sigma}^{\dagger} c_{\mathbf{k}, b, \sigma}+\sum_{b, \mathbf{k}} \Delta_{b}\left(c_{-\mathbf{k}, b, \downarrow}^{\dagger} c_{\mathbf{k}, b, \uparrow}^{\dagger}+\text { H.c. }\right) .
$$

Here, the summation goes over those four bands that cross the Fermi energy $(b=1 \ldots 4)$. We assume further that the superconducting gaps take only two different values: in the $p_{x, y}$ bands, $\Delta_{x y} \approx 7.5 \mathrm{meV}$, while for the $p_{z}$ bands, it is $\Delta_{z}$ $\approx 2.5 \mathrm{meV}$. In our work, we shall neglect furthermore the position dependence of the gaps around the magnetic impurity. This approximation is justified by the short coherence length in $\mathrm{MgB}_{2}$, as already explained in Sec. I.

\section{B. Interaction with a magnetic impurity}

To carry out a quantitative analysis of the magnetic impurity problem, we first need to establish how magnetic spins couple to the conduction band. The interaction part of the Hamiltonian depends on the specific location and electronic structure of the magnetic impurity considered. In what follows, we provide a detailed analysis for Mn impurities, which have already been doped into $\mathrm{MgB}_{2}$, although similar considerations hold for other types and positions of magnetic impurities. Mn ions presumably substitute the $\mathrm{Mg}$ atoms, and most likely take an $\mathrm{Mn}^{2+}$ configuration with a half-filled $d$ shell and a spin $S \approx 5 / 2 .{ }^{23}$ As shown in Fig. 2, the fivefold degeneracy of the $d$ states is lifted by the local hexagonal crystal field into three multiplets that we can label by the original angular momentum quantum numbers $\mu$ of the $d$ states, $|\mu\rangle$. Each of these states is occupied by a single electron and hybridizes through a hybridization $V_{\mu}$, with a specific local combination of $p$ states, $\psi_{\mu}$, that we construct next.

The $\mathrm{Mn}$ ion is in the middle of a cage of $12 \mathrm{~B}$ ions, which we shall label by the indices $i=1, \ldots, 12$. To start with, let us first construct the local hopping Hamiltonian between the Mn $d$ orbitals and the $p$ orbitals of a neighboring $\mathrm{B}$ ion " $i$ " at position $\mathbf{r}_{i}$. Let us now take a reference frame with the $\mathrm{Mn}$ in the origin and the $z$ axis pointing along the direction $\mathbf{n}_{i}$ of this neighboring ion. In this reference frame, with a good approximation, only the $\tilde{L}_{z}=0$ state of the five Mn $d$ states hybridizes with the $\widetilde{L}_{z}=0$ state of the B $p$ orbital. Correspondingly, the hybridization between the impurity and this neighbor can be approximated as

$$
V_{i}=V \Psi_{\mathbf{r}_{i}, \sigma}^{\dagger \|} d_{i, \sigma}^{\|}+\text {H.c., }
$$

where $d_{i, \sigma}^{\|}$is the annihilation operator for a local Mn $d$ orbital at the origin oriented along the direction $\mathbf{n}_{i}$. Similarly, $\Psi_{\mathbf{r}_{i}, \delta_{i}, \sigma}^{\|}$is the annihilation operator for the $p$ state at the B site oriented along the same direction. These operators are related to the operators occurring the $H_{0}$ by simple rotations,

$$
\Psi_{\mathbf{r}_{i}, \sigma}^{\|}=\sum_{\beta=x, y, z} \Psi_{\mathbf{r}_{i}, \beta, \sigma} n_{i}^{\beta}
$$

and

$$
d_{i, \sigma}^{\|}=\sum_{\mu} \alpha_{\mu}\left(\theta_{i}\right) e^{-i \widetilde{\phi}_{i}^{\mu}} d_{\mu, \sigma},
$$

where $d_{\mu, \sigma}$ refers to states with a quantization axis perpendicular to the B planes, $\widetilde{\phi}_{i}=\phi_{i}-\phi_{1}$, and

$$
\alpha_{\mu}(\theta)=\left(\begin{array}{c}
-\frac{1}{2} \sqrt{\frac{3}{2}} \sin ^{2} \theta \\
-i \frac{1}{2} \sqrt{\frac{3}{2}} \sin 2 \theta \\
\frac{1}{4}(1+3 \cos 2 \theta) \\
-i \frac{1}{2} \sqrt{\frac{3}{2}} \sin 2 \theta \\
-\frac{1}{2} \sqrt{\frac{3}{2}} \sin ^{2} \theta
\end{array} \mid .\right.
$$

Summing over all neighboring atoms and expressing all operators $\Psi_{\mathbf{r}_{i}, \beta, \sigma}$ in terms of the band operators $c_{\mathbf{k}, b, \sigma}$, we then obtain the following hybridization Hamiltonian,

$$
H_{V}=\sum_{b, \mu, \sigma} V_{b}^{(\mu)}\left(\Psi_{b, \mu, \sigma}^{\dagger} d_{\mu, \sigma}+d_{\mu, \sigma}^{\dagger} \Psi_{b, \mu, \sigma}\right),
$$

where the operator $\Psi_{b, \mu, \sigma}$ creates an electron with the same local $d$-state symmetry as $|\mu\rangle$ in band $b$, and can be expressed as 


$$
\begin{gathered}
\Psi_{b, \mu, \sigma}=\frac{1}{\sqrt{\Omega}} \sum_{\mathbf{k}} \tilde{f}_{\mu, b}(\mathbf{k}) c_{\mathbf{k}, b, \sigma}, \\
\tilde{f}_{\mu, b}(\mathbf{k})=\sum_{i, \alpha} \frac{\alpha_{\mu}^{*}}{A_{\mu, b}}\left(\theta_{i}\right) e^{i \tilde{\phi}_{i} \mu} n_{i}^{\alpha} e_{b ; \alpha, \delta_{i}}(\mathbf{k}) e^{i \mathbf{k} R_{i}},
\end{gathered}
$$

and $V_{b}^{(\mu)}=V A_{\mu, b}$. In these expressions, the normalization factor $A_{\mu, b}$ has been determined numerically and is defined by the condition that $\tilde{f}_{\mu, b}(\mathbf{k})$ be normalized at the Fermi surface

$$
\frac{1}{S_{b}} \int_{S_{b}} d^{2} \mathbf{k} \tilde{f}_{\mu, b}(\mathbf{k}) \tilde{f}_{\mu^{\prime}, b}^{*}(\mathbf{k})=\delta_{\mu, \mu^{\prime}} .
$$

Symmetry further implies that states belonging to the same irreducible representation have the same hybridization: $V_{b}^{(\mu)}$ $=V_{b}^{(-\mu)}$

The above hybridization Hamiltonian generates an effective exchange interaction between the Mn spin and the conduction electrons in the $\mathrm{B}$ bands, since it generates charge fluctuations to the $\mathrm{Mn}^{1+}$ and $\mathrm{Mn}^{3+}$ states. Second order perturbation theory in the hybridization leads to the effective exchange Hamiltonian:

$$
H_{\mathrm{int}}=\sum_{b, b^{\prime}, \mu, \alpha, \beta} \frac{1}{2} J_{\mu}^{b b^{\prime}} \Psi_{b, \alpha}^{\dagger} \boldsymbol{\sigma}_{\alpha \beta} \cdot \mathbf{S} \Psi_{b^{\prime}, \beta},
$$

where $\boldsymbol{\sigma}$ denotes the Pauli matrices, $\mathbf{S}$ is the $\mathrm{Mn}$ spin, and the exchange couplings are given by

$$
J_{\mu}^{b b^{\prime}} \propto \frac{V_{b}^{(\mu)} V_{b^{\prime}}^{(\mu)}}{\Delta E}=\frac{V^{2}}{\Delta E} A_{\mu, b} A_{\mu, b^{\prime}},
$$

with $\Delta E$, the characteristic energy of charge fluctuations. Note that the symmetry index $\mu$ is conserved in Eq. (14), thus there are five independent orbital channels of the conduction electrons that couple to the impurity spin. This is simple to understand on physical grounds: the half-filled $d$ shell has no orbital structure. Therefore, a conduction electron that arrives in an orbital state $\mu$ must be scattered back to the same orbital channel. However, electrons can be scattered between different conduction bands, and it is only their orbital label that is conserved over the scattering process. Therefore, in the absence of superconductivity, the channel labels play no special role, and the $S=5 / 2$ spin of the Mn ion would be exactly screened, resulting in a Fermi liquid state. ${ }^{9}$

By construction, the exchange couplings above satisfy $J_{\mu}^{b b^{\prime}}=\sqrt{J_{\mu}^{b b} J_{\mu}^{b^{\prime} b^{\prime}}}$, and furthermore, they are equal in channels $\pm \mu$ by symmetry. From Eq. (15), it also follows that all the results depend only on a single dimensionless coupling proportional to $V^{2} / \Delta E$. We define this coupling as

$$
g \equiv \frac{1}{5} \sum_{\mu, b} \varrho_{b} J_{\mu}^{b b},
$$

with $\varrho_{b}$ the density of states at the Fermi energy in band $b$ for one spin direction. Furthermore, in the rest of this paper, we shall only consider the classical limit $S \rightarrow \infty$, with $J_{m}^{b b^{\prime}} S$ =finite. In this limit, the impurity has no dynamics and we can solve the problem exactly.

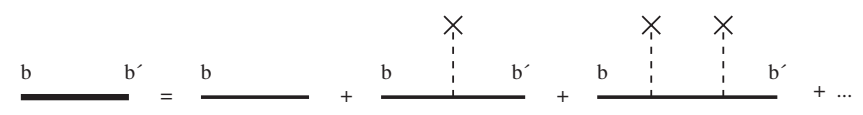

FIG. 3. Diagrammatic expansion for the Green's function when multiple scattering on the impurity site is considered. The solid line represents the full Green's function, and the thick line represents the noninteracting part of the Green's function. Each cross represents a scattering on an impurity site, and the dotted line stands for the impurity scattering potential. $b$ and $b^{\prime}$ stand for band indices.

To close this subsection, let us introduce Nambu spinors $\Phi_{\mathbf{k}, b}=\left\{\Phi_{\mathbf{k}, b}^{\tau \sigma}\right\}$ (Ref. 24) and

$$
\Phi_{\mathbf{k}, b} \equiv\left(\begin{array}{c}
c_{\mathbf{k}, b, \uparrow} \\
c_{\mathbf{k}, b, \downarrow} \\
-c_{-\mathbf{k}, b, \downarrow}^{\dagger} \\
c_{-\mathbf{k}, b, \uparrow}^{\dagger}
\end{array}\right) .
$$

The introduction of these spinors shall simplify our calculation considerably in the following sections. We can rewrite the Hamiltonian in terms of these in a compact form,

$$
\begin{aligned}
H= & \sum_{\mathbf{k}, b} \Phi_{\mathbf{k}, b}^{\dagger}\left(\hat{\varepsilon}_{\mathbf{k}, b} \tau^{z}+\hat{\Delta}_{b} \tau^{x}\right) \Phi_{\mathbf{k}, b} \\
& +\sum_{\mathbf{k}, \mathbf{k}^{\prime}, b, b^{\prime}, \mu} \frac{1}{2} J_{\mu}^{b b^{\prime}} \tilde{f}_{\mu, b}^{*}(\mathbf{k}) \Phi_{\mathbf{k}, b}^{\dagger} \boldsymbol{\sigma} \cdot \mathbf{S} \Phi_{\mathbf{k}^{\prime}, b^{\prime}} \tilde{f}_{\mu, b^{\prime}}\left(\mathbf{k}^{\prime}\right),
\end{aligned}
$$

where the $\tau$ 's denote Pauli matrices acting in the pseudospin (charge) index of the Nambu spinor. In the course of the derivation, we made use of time reversal symmetry that implies $\tilde{f}_{-\mu, b}(-\mathbf{k})=(-1)^{\mu} \tilde{f}_{\mu, b}^{*}(\mathbf{k})$, and doubled the Hilbert space so that the components of the Nambu spinors in Eq. (18) must be considered as independent variables.

\section{GREEN'S FUNCTION FORMALISM}

In this section, we shall discuss how the above Hamiltonian can be treated within the Green's function formalism. In the classical limit, the interaction with the impurity in Eq. (18) reduces to a spin-dependent potential scattering and, as we show below in detail, the problem can be solved exactly.

In the noninteracting case, $J_{\mu}^{b b^{\prime}}=0$, the Green's function is given by:

$$
G_{b}^{(0)}(\mathbf{k}, \omega)=\frac{1}{i \omega-\hat{\varepsilon}_{\mathbf{k}, b} \tau^{z}-\hat{\Delta} \tau^{x}},
$$

and it is a $16 \times 16$ matrix, diagonal in the band indices. In this expression, $\hat{\varepsilon}_{\mathbf{k}, b}$ and $\hat{\Delta}$ are also diagonal in band indices.

In the presence of impurity scattering, we can treat the scattering perturbatively and use multiple scattering theory to sum up the series to all orders. The diagrammatic expansion of the Green's function is represented in Fig. 3. In the first order of perturbation theory, the self-energy is given by 


$$
\Sigma^{(1)}\left(\mathbf{k}, \mathbf{k}^{\prime}, \omega\right)=\sum_{\mu} \widetilde{f}_{\mu}^{*}(\mathbf{k}) \frac{1}{2} J_{\mu}(\boldsymbol{\sigma} \cdot \mathbf{S}) \tilde{f}_{\mu}\left(\mathbf{k}^{\prime}\right)
$$

and is independent of the energy $\omega$. Here, we deliberately separated the form factors from the rest of the expression. The next order contribution gives

$$
\begin{aligned}
\Sigma_{b b^{\prime}}^{(2)}\left(\mathbf{k}, \mathbf{k}^{\prime}, \omega\right)= & \sum_{\mu, \mu^{\prime}, b^{\prime \prime}, \mathbf{q}} \hat{C}_{b b^{\prime \prime}}^{\mu}(\mathbf{k}, \mathbf{q})(\boldsymbol{\sigma} \cdot \mathbf{S}) \\
& \times G_{b^{\prime \prime}}^{(0)}(\mathbf{q}, \omega)(\boldsymbol{\sigma} \cdot \mathbf{S}) \hat{C}_{b^{\prime \prime} b^{\prime}}^{\mu}\left(\mathbf{q}, \mathbf{k}^{\prime}\right),
\end{aligned}
$$

where we introduced the notation $\hat{C}_{b b^{\prime}}^{\mu}(\mathbf{k}, \mathbf{q})$ $=1 / 2 \tilde{f}_{\mu, b}^{*}(\mathbf{k}) J_{\mu}^{b b^{\prime}} \tilde{f}_{\mu, b^{\prime}}(\mathbf{q})$. After summing over the momentum (as explained in Appendix B) and using the orthogonality of the form factor $\tilde{f}$ at the Fermi surface, we end up with the following expression:

$$
\sum_{b b^{\prime}}^{(2)}\left(\mathbf{k}, \mathbf{k}^{\prime}, \omega\right)=\sum_{\mu} \tilde{f}_{\mu, b}^{*}(\mathbf{k})\left[\frac{1}{2} \hat{J}_{\mu} \mathbf{S} \hat{F}(\omega) \frac{1}{2} \hat{J}_{\mu} \mathbf{S}\right]_{b b^{\prime}} \tilde{f}_{\mu, b^{\prime}}\left(\mathbf{k}^{\prime}\right)
$$

where $\hat{F}(\omega)$ denotes the matrix

$$
F_{b b^{\prime}}(\omega)=\delta_{b b^{\prime}} \varrho_{b} \int_{-D}^{D} d \varepsilon \frac{1}{\omega-\varepsilon \tau^{z}-\Delta_{b} \tau^{x}}
$$

with $D$ a high-energy cutoff that can be removed in the end of the calculation.

Higher order terms can be handled in a similar way. The final expression for Green's function is simply

$$
\begin{aligned}
G_{b b^{\prime}}\left(\mathbf{k}, \mathbf{k}^{\prime}, \omega\right)= & \delta_{\mathbf{k}, \mathbf{k}^{\prime}} \delta_{b b^{\prime}} G_{b}^{(0)}(\mathbf{k}, \omega) \\
& +G_{b}^{(0)}(\mathbf{k}, \omega) \sum_{\mu} \frac{1}{\Omega} \widetilde{f}_{\mu, b}^{*}(\mathbf{k})\left[\hat{T}_{\mu}(\omega)\right]_{b b^{\prime}} \\
& \times \widetilde{f}_{\mu, b^{\prime}}\left(\mathbf{k}^{\prime}\right) G_{b^{\prime}}^{(0)}\left(\mathbf{k}^{\prime}, \omega\right) .
\end{aligned}
$$

By the orthogonality relation [Eq. (13)], the quantum number $\mu$ is conserved. Therefore, the $T$ matrix $\hat{T}_{(\mu)}$ can be computed independently for each channel $\mu$ and is given by the following expression:

$$
\hat{T}_{\mu}(\omega)=\hat{J}_{\mu} \mathbf{S} \cdot \sigma / 2\left[1-\hat{F}(\omega) \hat{J}_{\mu} \mathbf{S} \cdot \sigma / 2\right]^{-1},
$$

where $\hat{F}(\omega)$ denotes the diagonal matrix $\hat{F}(\omega)=\hat{F}_{b b^{\prime}} \delta_{b b^{\prime}}$. Note that $\hat{F}(\omega)$ is diagonal in the spin labels. Therefore, even order terms in the $T$ matrix are spin independent. These terms can therefore be referred to as the "charge scattering channel." Odd order terms, on the other hand, give spindependent contributions and can be referred to as a "spin channel." The even (charge) channel can be directly resolved using STM technique, while for experimental observation of the odd (spin) channel contributions, spin-resolved STM is needed.

Impurity bound states and resonances can be identified from the pole structure of the $T$ matrices: True bound states correspond to zeroes of the determinants $\operatorname{det}\left\{\hat{T}_{\mu}^{-1}(\omega)\right\}$ on the real axis, and must satisfy $|\omega|<\Delta_{b}$ for all bands. Zeroes in the vicinity of the real axis, on the other hand, correspond to resonances. We found that each channel generates a bound state, but two of them are doubly degenerate by symmetry $(\mu \rightarrow-\mu)$.

It is, in general, impossible to find the poles of the $\hat{T}$ matrix analytically, and numerical calculations are needed. However, it is generally accepted that, at least phenomenologically, superconductivity in $\mathrm{MgB}_{2}$ can be explained using a two-band model. With this simple assumption, the positions of the resonances are given by the following equation:

$$
\left[1-g_{\mu}^{11} \alpha_{1}( \pm E)\right]\left[1-g_{\mu}^{22} \alpha_{2}( \pm E)\right]=\left(g_{\mu}^{12}\right)^{2} \alpha_{1}( \pm E) \alpha_{2}( \pm E),
$$

where $g_{\mu}^{b b^{\prime}} \equiv \pi S \sqrt{\varrho_{b} \varrho_{b}} J_{\mu}^{b b^{\prime}} / 2$ denotes the dimensionless couplings in channel $\mu$ and

$$
\alpha_{b}(\omega)=\left(\frac{\Delta_{b}+\omega}{\Delta_{b}-\omega}\right)^{1 / 2} .
$$

In the present case, these equations further simplify due to the relation $g_{\mu}^{11} g_{\mu}^{22}=\left(g_{\mu}^{12}\right)^{2}$ to

$$
g_{\mu}^{11} \alpha_{1}( \pm E)+g_{\mu}^{22} \alpha_{2}( \pm E)=1 .
$$

In the limiting case of $J_{\mu}^{12}=0$, Eq. (26) would give rise to two pairs of Shiba states ${ }^{1}$ for each channel $\mu$, corresponding to two independent bands. Exchange coupling between the two bands, however, removes half of these resonances. Similarly, in the realistic situation, we thus obtain five pairs of Shiba states corresponding to the five channels, but two pairs of them are twofold degenerate because of the symmetry $J_{\mu}^{b b^{\prime}}$ $=J_{-\mu}^{b b^{\prime}}$.

\section{DENSITY OF STATES}

Our main purpose is to compute the local tunneling density of states (LDOS) and the spin-resolved density of states near a magnetic impurity for various geometries. To obtain a quantitative estimate for the STM spectra, we performed a lengthy but straightforward tight-binding calculation to determine numerically the form factors $\tilde{f}_{\mu, b}(\mathbf{k})$, the exchange couplings, and the electronic wave functions above in various geometries.

The differential conductivity $d I / d V$ measured by STM is proportional with the local density of states, which can be calculated as the imaginary part of the retarded position dependent local Green's function:

$$
\begin{gathered}
\varrho_{c, \alpha}(\mathbf{r}, \omega)=-\frac{1}{2 \pi} \operatorname{Im} \operatorname{Tr}\left\{G\left(\mathbf{r}, p_{\alpha}, \omega\right) \frac{1+\tau_{z}}{2}\right\}, \\
G\left(\mathbf{r}, p_{\alpha}, \omega\right)=\frac{1}{\Omega} \sum_{\mathbf{k}, \mathbf{k}^{\prime}, b, b^{\prime}} e^{-i\left(\mathbf{k}-\mathbf{k}^{\prime}\right) \mathbf{R}} e_{b, \alpha \delta}^{*} e_{b, \alpha \delta} G_{b, b^{\prime}}\left(\mathbf{k}, \mathbf{k}^{\prime}, \omega\right) .
\end{gathered}
$$

Similar to the charge density of states, we can also define the spin density of states as 


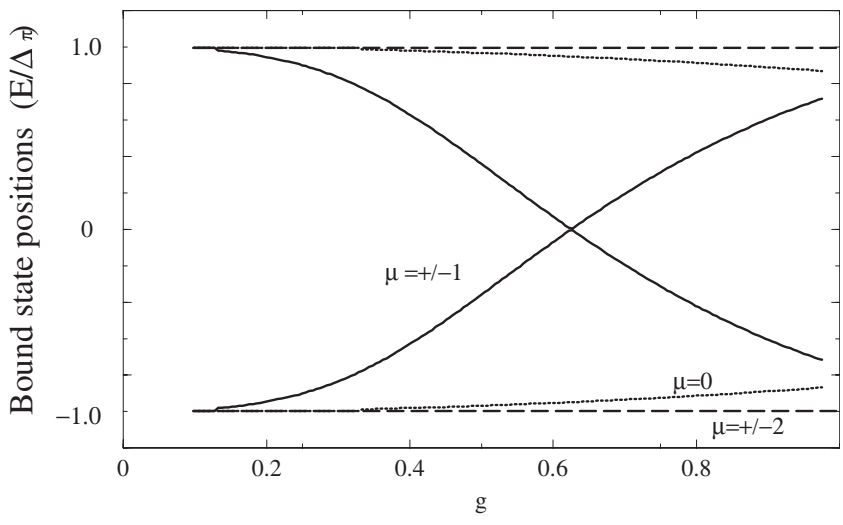

FIG. 4. Position of the quasiparticle poles for separate channels $\mu$. The resonances corresponding to the $\mu= \pm 1$ channels are well resolved for any value of the coupling strength $g$ (solid lines). For large enough $g(g>0.6)$ the $\mu=0$ resonance moves inside the gap (dotted lines). The couplings corresponding to the $\mu= \pm 2$ channels are much smaller that those in the other channels so the resonances for $\mu= \pm 2$ channels are still merged with the superconducting peaks at energy $\Delta_{\pi}$ (dashed lines).

$$
\varrho_{s, \alpha}(\mathbf{r}, \omega)=-\frac{1}{2 \pi} \operatorname{Im} \operatorname{Tr}\left\{G\left(\mathbf{r}, p_{\alpha}, \omega\right) \boldsymbol{\sigma} \mathbf{n} \frac{1+\tau_{z}}{2}\right\},
$$

where $\mathbf{n}$ is a unit vector pointing in the direction along which we measure the spin density of states.

The equations above refer to the case where the impurity is embedded in the bulk. However, both $\varrho_{c, \alpha}$ and $\varrho_{s, \alpha}$ can be computed easily from the analog of Eq. (24) for other boundary conditions too once the wave functions appearing in Eq. (3) are known. In the following subsections, we first compute the LDOS for an impurity in the bulk. Then we study the effect of a semi-infinite half-plane with the Mn impurity above and below the first B layer.

\section{A. Impurity in the bulk}

As a first step, we identify the positions of the resonances for each channel separately from the poles of the $\hat{T}$ matrix. In Fig. 4, we show the positions of the bound states and resonances obtained as a function of the dimensionless coupling $g$. The corresponding normalized $p_{z}$ LDOS at the B sites next to the Mn impurity is presented in Fig. 5 for different values of $g$. Due to hexagonal symmetry, all B sites around the magnetic impurity have the same LDOS. For small values of $g$, the bands are slightly interacting and only the most strongly coupled $\mu= \pm 1$ channels give rise to well resolved resonances in the gap for $g \leq 0.4$. Increasing the coupling $g$, the bands are more strongly interacting and the resonances corresponding to the $\mu=0$ channel move inside the gap too. This is accompanied, on one hand, by a transfer of weight between resonances and second by a shift in position of each resonance. We also observed small features at energies $\omega$ $=7.5 \mathrm{meV}$, i.e., at the energy corresponding to $\Delta_{\sigma}$, due to the coupling between the bands (not shown in this figure).

Figure 5 also shows the density of states at the nextnearest-neighbor sites. The wave functions of the Shiba states and thus the amplitudes of the corresponding reso-
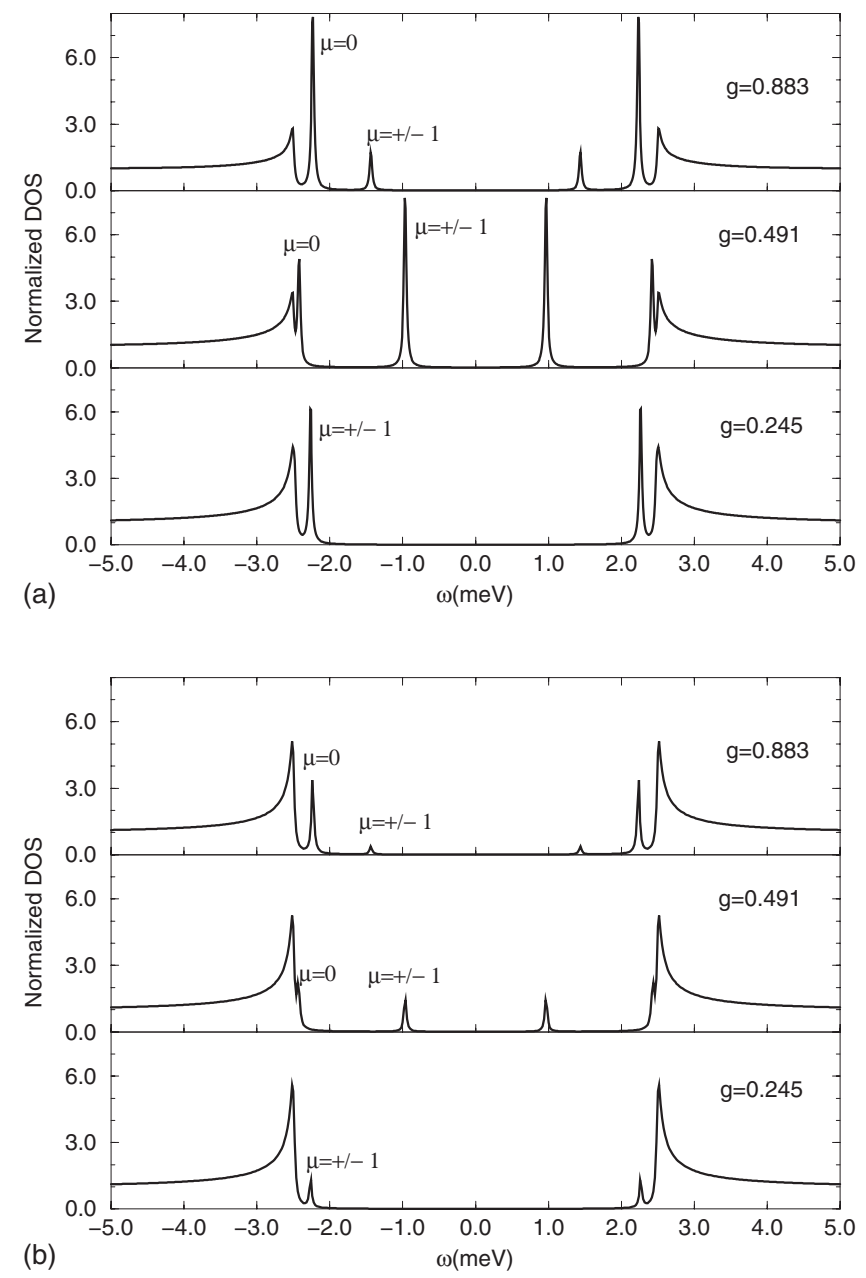

FIG. 5. Upper panel: Normalized LDOS at the nearest-neighbor boron sites (labeled A in Fig. 2) for the $p_{z}$ orbitals, for different coupling constants. Lower panel: Normalized LDOS at the nextnearest neighbor boron sites labeled B in Fig. 2.

nances in the spectrum depend a lot on the tunneling position: The weight and the amplitude of the resonances decrease considerably while their position remains unchanged. This suppression reflects the local structure of Shiba states. At the same time, the coherence peaks near the superconducting gap edge gain some spectral weight, but they are still quite reduced compared to the bulk. Further away from the impurity site, the superconducting coherence peaks are completely restored and the bound states have negligible amplitudes. For generic values of the exchange coupling, usually two well-separated pairs of resonances can be observed, corresponding to the $\mu= \pm 1$ and $\mu=0$ channels. The exchange couplings in channels $\mu= \pm 2$ are much smaller than those in channels $\mu= \pm 1$ and $\mu=0$, and therefore, the corresponding bound states are merged with the superconducting coherence peak.

The Shiba states are also strongly spin polarized, as is obvious from the spin polarization in the local density of states shown in Fig. 6. This fact has an important consequence from the point of view of the observability of these bound states. As always, a sharp local spectroscopic feature could be difficult to detect if it is overshadowed by the in- 


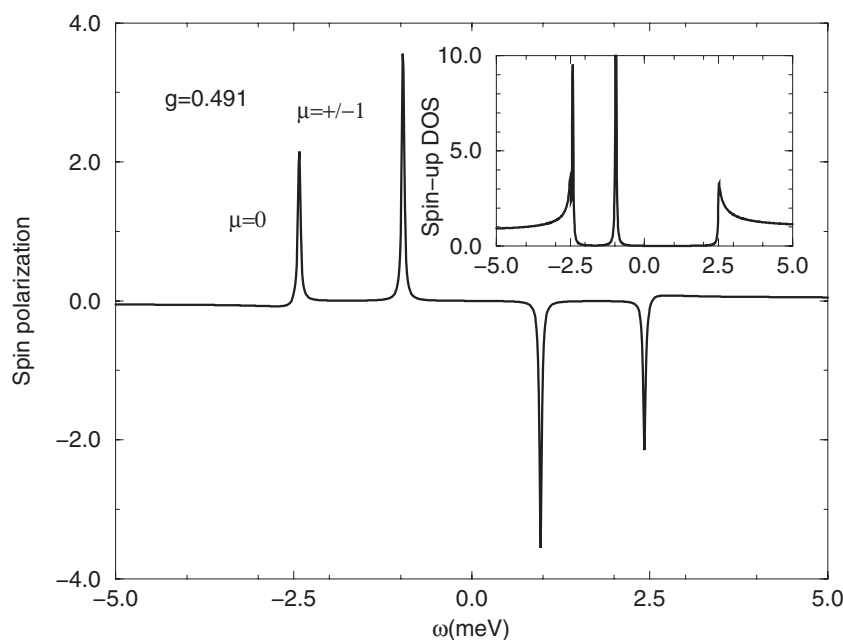

FIG. 6. Spin polarization (odd part of the spectrum and spin-up density of states for Site A in Fig. 2).

tense continuous background of the superconductor. However, the background continuum in a superconductor is not, generally speaking, spin polarized. Thus, even if a Shiba peak happens to be close to one of the otherwise dominant BCS coherence peaks, a spin-polarized STM can distinguish the Shiba states from the continuum, ${ }^{25}$ since the asymmetric part of the spin-polarized spectrum has sharp peaks at the resonances but is predicted to be featureless otherwise. Therefore, spin-polarized STM is clearly an ideal tool to identify the multiple Shiba states.

\section{B. Impurity in the vicinity of a surface}

As we mentioned already, the effect of a surface can be taken into account by simply modifying the wave functions that appear in the expansion of the operators $\Psi_{\mathbf{r}, \alpha, \sigma}$,

$$
\Psi_{\mathbf{r}, \alpha, \sigma}=\sum_{\mathbf{k}_{\perp}, k_{z}, b} \varphi_{\mathbf{k}_{\perp}, k_{z}, b}(\mathbf{r}) c_{\mathbf{k}_{\perp}, k_{z}, b, \sigma} .
$$

Here, $\mathbf{k}_{\perp}$ is the in-plane momentum and $k_{z}$ is the momentum perpendicular to the surface. Note that the surface breaks translational symmetry along the $\hat{z}$ direction, and therefore, only $k_{z}>0$ values are permitted. The wave functions above must satisfy the appropriate boundary conditions and can be expressed within our tight-binding formalism as

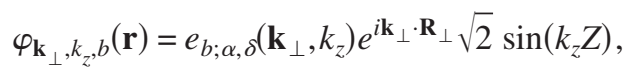

with $Z=0$ corresponding to the first layer in the vacuum.

Our calculations for an impurity in the bulk can easily be extended to this case as well with minor modifications. If the magnetic impurity is well inside the bulk, we recover the results discussed in the previous subsection. In Fig. 7 (upper panel), we represent the LDOS at nearest-neighbor B atoms for the case when the Mn impurity is below the top B layer. The amplitudes of the resonances are slightly reduced in this case compared to the bulk system, and also, the positions are modified due to the local density of states that is slightly modified in the vicinity of the surface. Moving away from the impurity, the weights of the resonances start to decrease
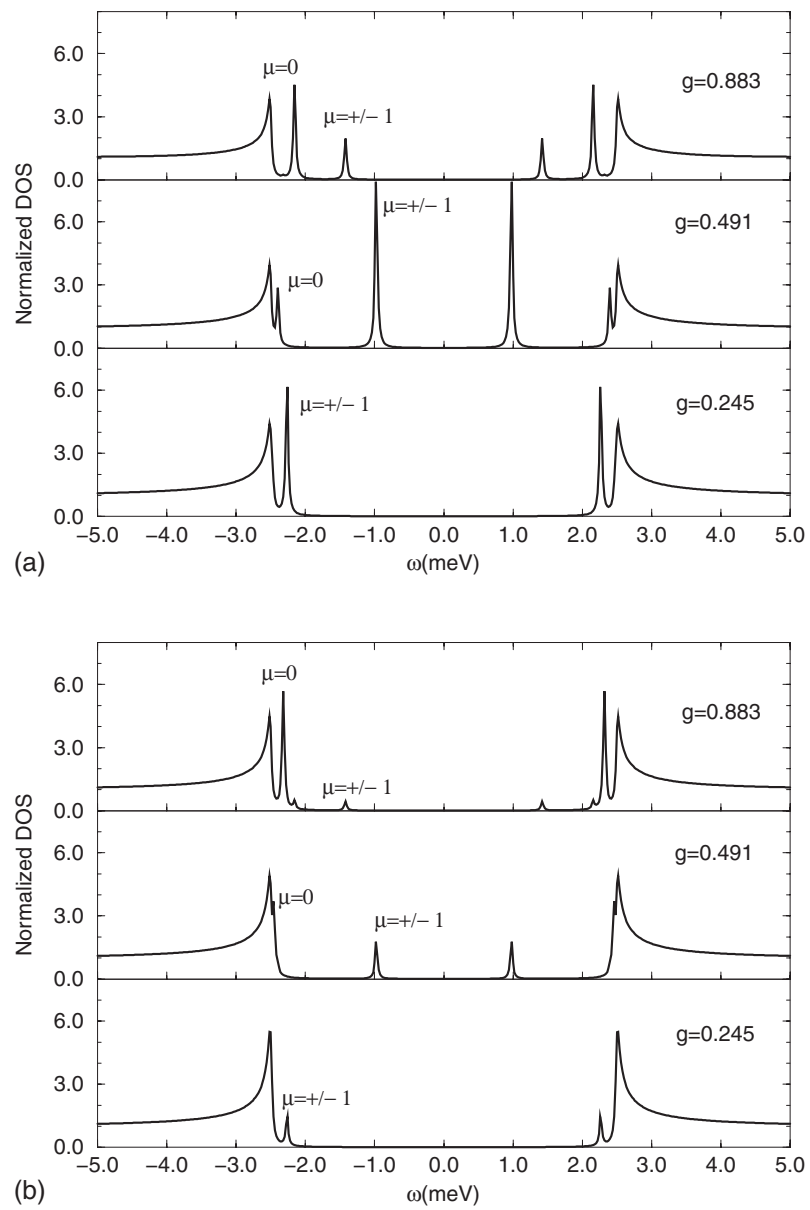

FIG. 7. Upper panel: Normalized density of states for site A as function of frequency for different values of $g$ in the geometry when the Mn impurity is below the first B layer. Lower panel: Normalized LDOS at the next-nearest-neighbor boron sites B of Fig. 2, when the Mn impurity is just below the first layer.

and the superconducting coherence peaks are gradually recovered. In this configuration, at sites more than two lattice constants away from the impurity site, the superconducting coherence peak is already completely recovered.

For spin-resolved scanning tunneling spectroscopy, the tunneling current can be separated into an unpolarized part $I_{0}$, which depends only on the LDOS, and a spin-polarized contribution $I_{p}$ given by the projection of the local magnetization density at the tunneling site onto the magnetization direction of the tip. The spin-polarized contribution to the local differential conductivity is therefore proportional to the magnetization density $d I_{p} / d V \propto P_{T} \cos \theta \varrho_{s}\left(\mathbf{r}_{i}, \omega=e V\right)$, where $P_{T}$ denotes the polarization of the tip, and $\theta$ is the angle between the magnetization axes of the tip and the impurity spin.

In Fig. 8, we present the local spin polarization at site A for $g=0.491$. For the same reasons as before, only the contribution of the $p_{z}$ orbital is shown. The relative orientation of the impurity spin and the tip can also be fixed by a small external magnetic field in these experiments. However, the angle $\theta$ is not arbitrary even in the absence of an external field, since in the vicinity of a ferromagnetic STM tip a magnetic impurity would be presumably aligned with the mag- 


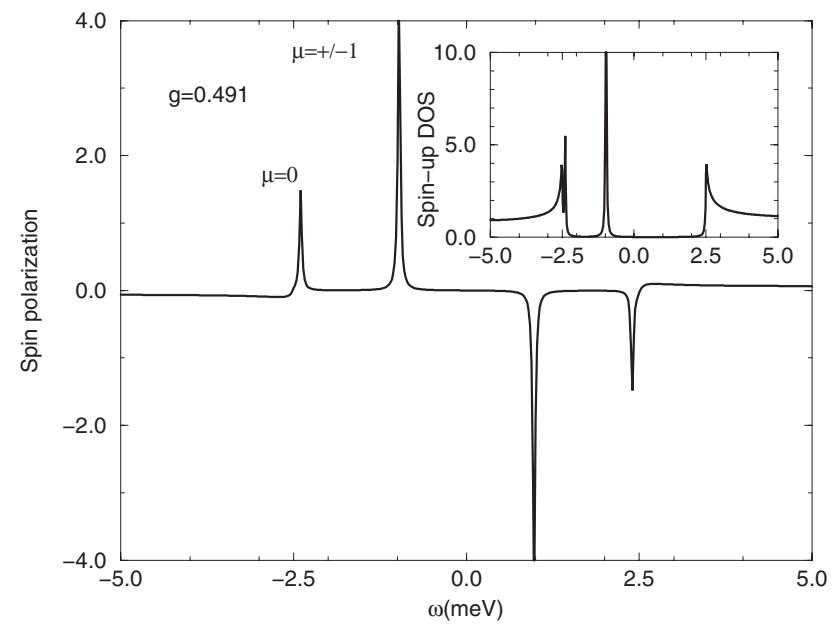

FIG. 8. Spin polarization and spin-resolved density of states for site A of Fig. 2.

netization of the tip due to stray fields. The most important feature we observe is a transfer of weight from states in the gap to states in the continuum due to the interband coupling through the magnetic impurity. The inset presents the total spin-polarized tunneling density of states for the same coupling $g$, for a complete polarization of the tip $P_{T}=1$, and a perfect alignment $\theta=0$.

\section{CONCLUSIONS}

We presented a detailed theoretical investigation of the effect of a single Mn magnetic impurity on the superconducting properties of $\mathrm{MgB}_{2}$. Our description is based on a microscopic model, which assumes nearest-neighbor hopping from the localized orbital of the Mn to the neighboring B orbitals. We have shown that a magnetic impurity generally induces multiple Shiba states in the electronic structure of $\mathrm{MgB}_{2}$. In particular, for Mn, we found five pairs of Shiba states in the gap, two of which were twofold degenerate. We have taken into account realistic band structure and the effect of surface states on the local spectrum. Our calculation of both conventional and spin-resolved STM $^{25}$ spectra near the impurity site showed that these states can be clearly resolved by both methods. Similar multiple Shiba states should appear in other superconductors due to the internal structure of the magnetic impurity.

It is intriguing to speculate what these local probes will eventually see in an actual experiment. Clearly, despite decades of pioneering investigation, local spectroscopy of spin impurity states in a superconductor still has the potential of revealing new features that have not yet been documented. For example, our calculations assume classical spin degrees of freedom, whereas the experimental measurements could reveal-besides a classical behavior-effects of screening of a quantum spin by the superconductor, leading to either a full screening or a reduction of the effective spin carried by the impurity. The quantitative discussion of such effects goes beyond the scope of the present paper. Nevertheless, our calculations will provide an important benchmark for comparison to experiments, a benchmark that includes the presence of multiple channels of scattering.

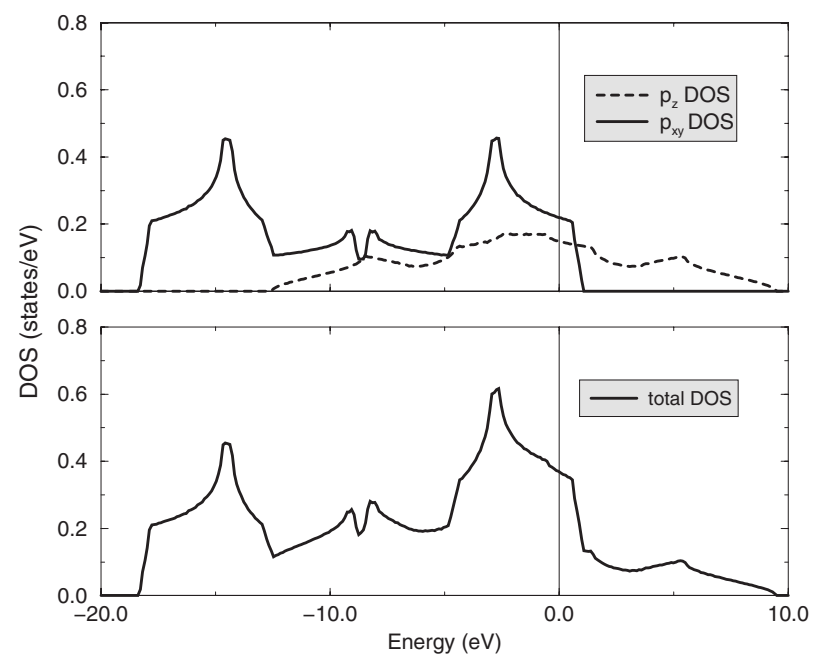

FIG. 9. Top: Density of states for $p_{x, y}$ and $p_{z}$ bands. Bottom: Total density of states. The vertical line represents the position of the Fermi energy.

The results we obtained in this paper are relevant and relatively easy to generalize for other compounds. For example, recent STM measurements have focused on Ti impurities in another multiband superconductor $\mathrm{Sr}_{2} \mathrm{RuO}_{4}$. While these experimental results are preliminary as the magnetization state of $\mathrm{Ti}$ is not clear, and there are several differences between $\mathrm{MgB}_{2}$ and $\mathrm{Sr}_{2} \mathrm{RuO}_{4}$, it is clear that our framework provides a suitable platform for studying $\mathrm{Sr}_{2} \mathrm{RuO}_{4}$ as well. As we mentioned above, $\mathrm{MgB}_{2}$ crystallizes in the hexagonal $\mathrm{AlB}_{2}$-type structure, ${ }^{13}$ and the band structure of $\mathrm{MgB}_{2}$ is also somewhat peculiar. Nevertheless, as shown in the seminal paper of Nozières and Blandin, ${ }^{9}$ although the form of the exchange Hamiltonian depends a lot on the specific material and point group considered, in most cases, similar to Mndoped $\mathrm{MgB}_{2}$, several channels of conduction electrons couple to the local impurity degrees of freedom and result in multiple Shiba states. Therefore, the appearance of multiple Shiba states is a rather general phenomenon.

An interesting result of our analysis is that, although it may be difficult to resolve a Shiba state close to the coherence peak with conventional STM methods, the antisymmetrical part of a spin-resolved STM clearly separates these states in the STM spectrum. The weight of a given pair of Shiba states may, however, be very sensitive to the particular atomic state into which electrons tunnel from the STM tip, and depends also on the precise position of the tip.

\section{ACKNOWLEDGMENTS}

We are grateful to D. Agterberg, G. Crabtree, J. C. Seamus Davis, M. Iavarone, G. Karapetrov, I. Mazin, K. Tanaka, A. Yazdani, and J. Zasadzinski for useful discussions. This work was supported by the U. S. Dept. of Energy, Office of Science, under Contract No. W-31-109-ENG-38, Hungarian Grants No. OTKA NF061726, No. T046267, and No. K73361, and Romanian Grant No. CNCSIS 1/780/2007. B. J. was also supported by NSF-NIRT Grants DMR02-10519 and ECS-0609249, and the Alfred P. Sloan Foundation. 


\section{APPENDIX A: TIGHT-BINDING HAMILTONIAN}

In this Appendix, we present the basic results obtained from the tight-binding analysis of the bulk system. The matrix elements of the Hamiltonian given in Eq. (4) are given by

$$
\begin{gathered}
H_{x, 1 ; x, 1}(\mathbf{k})=\epsilon_{x y}-2 t_{x y} \cos \left(k_{z} \frac{c}{a}\right), \\
H_{x, 1 ; x, 2}(\mathbf{k})=t_{\perp}+\left(\frac{3}{4} t_{\|}+\frac{1}{4} t_{\perp}\right) \exp \left(-i \frac{3}{2} k_{y}\right) 2 \cos \frac{\sqrt{3}}{2} k_{x}, \\
H_{x, 1 ; y, 2}(\mathbf{k})=-\frac{\sqrt{3}}{4}\left(t_{\|}-t_{\perp}\right) \exp \left(-i \frac{3}{2} k_{y}\right) 2 i \sin \frac{\sqrt{3}}{2} k_{x}, \\
H_{y, 1 ; y, 2}(\mathbf{k})=t_{\|}+\left(\frac{3}{4} t_{\perp}+\frac{1}{4} t_{\|}\right) \exp \left(-i \frac{3}{2} k_{y}\right) 2 \cos \frac{\sqrt{3}}{2} k_{x}, \\
H_{z, 1 ; z, 1}(\mathbf{k})=\epsilon_{z}-2 t_{z} \cos \left(k_{z} \frac{c}{a}\right), \\
H_{z, 1 ; z, 2}(\mathbf{k})=t\left\{\begin{array}{l}
\left.1+\exp \left[-i\left(\frac{\sqrt{3}}{2} k_{x}+\frac{3}{2} k_{y}\right)\right]\right\} .
\end{array}\right.
\end{gathered}
$$

All the other matrix components of the Hamiltonian matrix can be written in terms of those given in Eq. (A1) as follows: $H_{y, 1 ; y, 1}(\mathbf{k})=H_{x, 2 ; x, 2}(\mathbf{k})=H_{y, 2 ; y, 2}(\mathbf{k})=H_{x, 1 ; x, 1}(\mathbf{k}), \quad H_{y, 1 ; x, 2}(\mathbf{k})$ $=H_{x, 1 ; y, 2}(\mathbf{k}), H_{z, 2 ; z, 2}(\mathbf{k})=H_{z, 1 ; z, 1}(\mathbf{k})$. All the other elements are equal to zero. This matrix is Hermitian, $H_{j i}=H_{i j}^{*}$ for $i \neq j$. The best fit to other calculated band structure ${ }^{14}$ is obtained for the following set of parameters: $\epsilon_{x y}=-8.6 \mathrm{eV}, \epsilon_{z}=-1.5 \mathrm{eV}, t$ $=2.0 \mathrm{eV}, t_{z}=2.5 \mathrm{eV}, t_{\|}=4.5 \mathrm{eV}, t_{\perp}=1.8 \mathrm{eV}$, and $t_{x y}$ $=0.1 \mathrm{eV}$. In our calculation, $t$ and $t_{z}$ are the hopping integral corresponding to the $p_{z}$ orbitals: $t$ is the in-plane hopping between the nearest neighbors ( $\pi$ bonding) and $t_{z}$ is the out of plane hopping ( $\sigma$ bonding). The parameters $t_{\|}$and $t_{\perp}$ denote $\sigma$ and $\pi$-like hopping integrals for the in-plane $p_{x, y}$ orbitals. Finally, the out-of-plane hopping integral is given by $t_{x y}$. This parameter is very small, so there is practically no dispersion along the $\Gamma-A$ line.

The corresponding density of states has been calculated in the framework of the Green's function formalism as $\varrho(\omega)=$ $-\frac{1}{\pi} \sum_{\mathbf{k}} \operatorname{Im} G_{b}(\mathbf{k}, \omega)$, where $G_{b}(\mathbf{k}, \omega)$ is the Green's function corresponding to every band $b$. The resulting DOS is presented in Fig. 9. The values for the DOS at the Fermi surface for the bands that cross the Fermi surface are $\varrho_{x}$ $=0.081$ states $/ \mathrm{eV}, \quad \varrho_{y}=0.13$ states $/ \mathrm{eV}, \quad$ and $\varrho_{z 1}=\varrho_{z 2}$ $=0.75$ states $/ \mathrm{eV}$, in reasonable agreement with more sophisticated band structure calculations. ${ }^{14}$

\section{APPENDIX B: AVERAGE OVER THE FERMI SURFACE}

Throughout our analysis, we have to evaluate averages over the Fermi surface. For a given band we have to calculate

$$
\frac{1}{S_{b}} \int_{S_{b}} \varphi(\mathbf{k}) d^{2} \mathbf{k}
$$

where $S_{b}$ represents the Fermi surface area for band $b$ and $\varphi(\mathbf{k})$ is a momentum dependent function. The Fermi surface was obtained in our calculation by numerically solving the equation $\varepsilon_{\mathbf{k}, b}=0$. To evaluate Eq. (B1), we replace the integration over the Fermi surface with an integration over an energy shell of thickness $d \varepsilon$. First, the area of the Fermi surface can be calculated as

$$
S_{b}=\int_{S_{b}} d^{2} \mathbf{k}=\frac{1}{d \varepsilon} \int_{\text {shell }} d^{3} \mathbf{k}\left|\nabla \varepsilon_{\mathbf{k}, b}\right| .
$$

In a similar way, the average of any momentum-dependent function can be evaluated as

$$
\int_{S_{b}} \varphi(\mathbf{k}) d^{2} \mathbf{k}=\frac{1}{d \varepsilon} \int_{\text {shell }} d^{3} \mathbf{k}\left|\nabla \varepsilon_{\mathbf{k}, b}\right| \varphi(\mathbf{k}) .
$$

Our quantity is therefore given by the expression:

$$
\frac{1}{S_{b}} \int_{S_{b}} \varphi(\mathbf{k}) d^{2} \mathbf{k}=\frac{\int_{\text {shell }} d^{3} \mathbf{k}\left|\nabla \varepsilon_{\mathbf{k}, b}\right| \varphi(\mathbf{k})}{\int_{\text {shell }} d^{3} \mathbf{k}\left|\nabla \varepsilon_{\mathbf{k}, b}\right|} .
$$

In the numerical calculations, we used a $100 \times 100 \times 100$ discretization of the first Brillouin zone. For each site in the discretized lattice, we calculated the energy values corresponding to band $b$. We then tested if one of these $10^{6}$ cells overlapped with the shell of thickness $d \varepsilon$. If it did, we generated a mesh of $15 \times 15 \times 15$ within this cell to compute the cell's contribution to Eq. (B4).

In our calculations, the number of points around the Fermi surface was larger than $10^{6}$ within an energy shell of $10 \mathrm{meV}$. This was used to evaluate the average of the form factors and their Fourier transforms at the Fermi surface with a precision of $\sim 10^{-3}$.

\section{APPENDIX C: MOMENTUM SUMMATION}

In this section, we explain the method that we used to evaluate the momentum summation in the first Brillouin zone. During the calculations, we have to evaluate the expression of the form:

$$
\frac{1}{V} \sum_{\mathbf{k}} \varphi(\mathbf{k}) G_{b}^{(0)}(\mathbf{k}, \omega),
$$

where $\varphi(\mathbf{k})$ is a momentum dependent function (usually the form factor or a combination including form factors and other momentum-dependent functions) and $G_{b}^{(0)}(\mathbf{k}, \omega)$ is the free Green's function. The free Green's function depends on momentum only through the energy of the given band $\varepsilon_{\mathbf{k}, b}$. We approximated therefore the summation as

$$
\frac{1}{V} \sum_{\mathbf{k}} \varphi(\mathbf{k}) G_{b}^{(0)}(\mathbf{k}, \omega)
$$




$$
\rightarrow \varrho_{b} \int_{-\infty}^{\infty} d \varepsilon G_{b}^{(0)}(\varepsilon, \omega) \frac{1}{S_{b}} \int_{S_{b}} d^{2} \mathbf{k} \varphi(\mathbf{k})
$$

with $\varrho_{b}$ the density at the Fermi surface in band $b$. The integration of the Green's function over the energy can be done analytically and the result is simply

$$
F_{b}(\omega)=\varrho_{b} \int_{-\infty}^{\infty} d \varepsilon \frac{1}{\omega-\varepsilon \tau^{z}-\Delta_{b} \tau^{x}} .
$$

For $|\omega|<\Delta_{b}$, the function $F_{b}(\omega)$ has only real parts and simplifies to $F_{b}(\omega)=-\pi \varrho_{b}\left(\omega+\Delta_{b} \tau^{x}\right) /\left(\Delta_{b}^{2}-\omega^{2}\right)^{-1 / 2}$, while for $|\omega|>\Delta_{b}$, it is purely imaginary. The other term which represents an average over the Fermi surface was calculated numerically as explained in Appendix B.
${ }^{1}$ H. Shiba, Prog. Theor. Phys. 40, 435 (1968).

${ }^{2}$ A. Yazdani, B. A. Jones, C. P. Lutz, M. F. Crommie, and D. M. Eigler, Science 275, 1767 (1997).

${ }^{3}$ D. Poilblanc, D. J. Scalapino, and W. Hanke, Phys. Rev. Lett. 72, 884 (1994); J. M. Byers, M. E. Flatte, and D. J. Scalapino, ibid. 71, 3363 (1993).

${ }^{4}$ M. E. Flatté and D. E. Reynolds, Phys. Rev. B 61, 14810 (2000); M. E. Flatté and J. M. Byers, ibid. 56, 11213 (1997); Phys. Rev. Lett. 78, 3761 (1997).

${ }^{5}$ A. V. Balatsky, M. I. Salkola, and A. Rosengren, Phys. Rev. B 51, 15547 (1995); M. I. Salkola, A. V. Balatsky, and J. R. Schrieffer, ibid. 55, 12648 (1997).

${ }^{6}$ S. H. Pan, E. W. Hudson, K. M. Lang, H. Eisaki, S. Uchida, and J. C. Davis, Nature (London) 403, 746 (2000); E. W. Hudson, S. H. Pan, A. K. Gupta, K. W. Ng, and J. C. Davis, Science 285, 88 (1999).

${ }^{7}$ M. S. Choi, M. Lee, K. Kang, and W. Belzig, Phys. Rev. B 70, 020502(R) (2004).

${ }^{8}$ F. Siano and R. Egger, Phys. Rev. Lett. 93, 047002 (2004).

${ }^{9}$ P. Nozières and A. Blandin, J. Phys. (Paris) 41, 193 (1980).

${ }^{10}$ L. Bergman, D. Alexson, P. L. Murphy, R. J. Nemanich, M. Dutta, M. A. Stroscio, C. Balkas, H. Shin, and R. F. Davis, Phys. Rev. B 59, 12977 (2003).

${ }^{11}$ For a series of recent results and reviews see the articles in Physica C 466, 1 (2007).

${ }^{12}$ J. Nagamatsu, N. Nakagawa, T. Muranaka, Y. Zenitani, and J. Akimitsu, Nature (London) 410, 63 (2001).

${ }^{13}$ S. L. Bud'ko, G. Lapertot, C. Petrovic, C. E. Cunningham, N. Anderson, and P. C. Canfield, Phys. Rev. Lett. 86, 1877 (2001).

${ }^{14}$ J. Kortus, I. I. Mazin, K. D. Belashchenko, V. P. Antropov, and L. L. Boyer, Phys. Rev. Lett. 86, 4656 (2001).

${ }^{15}$ F. Giubileo, D. Roditchev, W. Sacks, R. Lamy, D. X. Thanh, J.
Klein, S. Miraglia, D. Fruchart, J. Marcus, and P. Monod, Phys. Rev. Lett. 87, 177008 (2001).

${ }^{16}$ P. Szabo, P. Samuely, J. Kacmarcik, Th. Klein, J. Marcus, D. Fruchart, S. Miraglia, C. Marcenat, and A. G. M. Jansen, Phys. Rev. Lett. 87, 137005 (2001).

${ }^{17}$ H. Schmidt, J. F. Zasadzinski, K. E. Gray, and D. G. Hinks, Phys. Rev. B 63, 220504(R) (2001).

${ }^{18}$ S. Tsuda, T. Yokoya, T. Kiss, Y. Takano, K. Togano, H. Kito, H. Ihara, and S. Shin, Phys. Rev. Lett. 87, 177006 (2001).

${ }^{19}$ R. S. Gonnelli, D. Daghero, G. A. Ummarino, A. Calzolari, M. Tortello, V. A. Stepanov, N. D. Zhigadlo, K. Rogacki, J. Karpinski, F. Bernardini, and S. Massidda, Phys. Rev. Lett. 97, 037001 (2006); D. Daghero, A. Calzolari, G. A. Ummarino, M. Tortello, R. S. Gonnelli, V. A. Stepanov, C. Tarantini, P. Manfrinetti, and E. Lehmann, Phys. Rev. B 74, 174519 (2006).

${ }^{20}$ K. Rogacki, B. Batlogg, J. Karpinski, N. D. Zhigadlo, G. Schuck, S. M. Kazakov, P. Wagli, R. Puzniak, A. Wisniewski, F. Carbone, A. Brinkman, and D. van der Marel, Phys. Rev. B 73, 174520 (2006).

${ }^{21}$ G. A. Ummarino, J. Supercond. Novel Magn. 20, 639 (2007).

${ }^{22}$ J. Kortus, Physica C 456, 54 (2007).

${ }^{23} \mathrm{~S}$. Xu, Y. Moritomo, K. Kato, and A. Nakamura, J. Phys. Soc. Jpn. 70, 1889 (2001).

${ }^{24}$ With this definition Nambu spinors have nice transformation properties, which allows us to express the total Hamiltonian in an especially simple and transparent form. [See, e.g., P. Coleman, E. Miranda, and A. Tsvelik, Phys. Rev. B 49, 8955 (1994).]

${ }^{25}$ See, for example, S. Heinze, M. Bode, A. Kubetzka, O. Pietzsch, X. Nie, S. Blugel, and R. Wiesendanger, Science 288, 1805 (2000). 